\title{
工程教育专业认证背景下的化工原理课程体系的建设与改革
}

陶彩虹*, 刘宝勇, 盛丽, 雷洋, 蔡洁琼

兰州交通大学化学与生物工程学院, 兰州 730070

摘要: 化工原理课程体系包括化工原理、化工原理实验和化工原理课程设计三大环节, 该课程体系对于培养工科学 生科学的工程思维方法和工程意识、提高其工程实践技能与工程素质都具有十分重要的意义。而工程素质与实践能 力是工程技术人员必须具备的重要能力。本文从三大环节入手, 分别探讨了工程教育认证和新工科建设背景下对化 工原理课程体系中的三门课程进行课程改革的思路和方法, 从根本上提高化工原理课程体系的教学质量, 培养真正 能解决工程实际问题的工程技术人才。

关键词: 化工原理; 教学方法; 专业认证

中图分类号: G64; O6; TQ01

\section{Construction and Reform of the Course System of Principles of Chemical Engineering under the Background of Engineering Education Certification}

\author{
Caihong Tao *, Baoyong Liu, Li Sheng, Yang Lei, Jieqiong Cai \\ School of Chemical and Biological Engineering, Lanzhou Jiaotong University, Lanzhou 730070, China.
}

\begin{abstract}
The curriculum system of principles of chemical engineering includes three segments: principles of chemical engineering, laboratory of principles of chemical engineering and curriculum design of principles of chemical engineering. This course is of great significance to guide students to establish cultivate scientific engineering thinking methods and engineering consciousness. It is also very important for improving students' engineering practice skills and engineering quality, the two essential abilities for engineering technicians. Starting from the above three segments, this paper discusses the methods of curriculum reform for three courses under the background of engineering education certification and new engineering construction, so as to fundamentally improve the teaching quality of chemical engineering principle curriculum system and train qualified engineering students who can really solve the practical problems of Engineering.
\end{abstract}

Key Words: Chemical engineering principle; Teaching method; Professional certification

高等教育的快速发展对一个国家综合国力的提高有很重要的作用, 工程教育与产业发展密不可 分。随着科学技术的快速发展, 对工程技术人才的知识和技能都提出了新要求 ${ }^{[1]}$ 。作为我国高等教 育重要组成部分的工程教育, 在高等教育体系中起着举足轻重的作用, 在世界上各个国家工业化的 进程中, 工程教育都发挥了不可替代的作用。新工业革命时代, 科技和工业发展的特征是 “快速变 化” 和 “加速变化”, 要求工程科技人才也要适应快速发展。高等教育中的传统培养模式、课程体系 和课程内容等需要进行改革和创新。工程教育专业认证是国际通行的工程教育质量保障制度, 也是

收稿: 2020-12-15; 录用: 2021-03-16; 网络发表: 2021-03-29

“通讯作者, Email: taoch@mail.lzjtu.cn

基金资助: 兰州交通大学 “百名青年优秀人才培养计划” 项目; 兰州交通大学思政教改专项; 兰州交通大学金课建设项目 
实现工程教育国际互认和工程师资格国际互认的重要基础 ${ }^{[2]}$ 。工程教育专业认证的核心就是要确认 工科专业毕业生达到行业认可的既定质量标准要求, 是一种以培养目标和毕业出口要求为导向的合 格性评价。工程教育专业认证要求专业课程体系设置、师资队伍配备、办学条件配置等都围绕学生 毕业能力达成这一核心任务展开, 并强调建立专业持续改进机制和文化以保证专业教育质量和专业 教育活力 ${ }^{[3]}$ 。

化工类专业是通用型过程工程专业, 也是与高新科技密切相关的工科专业。科技发展日新月异, 人们对化工产品的需求日渐多元化, 所以化工过程的复杂性、多样性趋势日趋明显, 这对化工类专 业学生的培养提出了更高的要求。学生应具备较强的工程实践能力和一定的创新能力, 尤其是要具 备解决复杂工程问题的能力。传统的教学模式一般是以教师为中心, 教学方法和方式相对比较单一, 教学资源的配置不够完善和合理, 对于学生上述能力的培养造成了一定的困难, 学生的能力达成度 普遍较低 ${ }^{[4]}$, 教学模式的改革迫在眉睫。化工原理课程体系是化工类及相关专业的核心课程, 有着 较强的理论与综合性, 旨在培养学生的综合应用能力, 在理论与实践的教学过程中也要顺应工程教 育专业认证和新工科建设的需要进行适时改变。要求学生不仅掌握课程的基本理论, 具有一定的运 用原理解决实际工程中复杂问题的能力, 同时还要具有良好的人文素养、高尚的道德情操 ${ }^{[5]}$ 。我校 化学工程与工艺、生物工程和过程装备与控制等专业都要在2020年前后申请进行认证, 在认证前需 对每个专业的培养方案和各个课程的教学大纲等进行修订, 并对各门课程的教学质量提出新的要求。 以此为契机, 我们对化工原理课程体系进行了相应的改革。工程教育认证的三点理论分别是本科学 生作为认证的中心、学生的就业和出路作为认证的引导、找到问题并解决问题作为认证的改进 ${ }^{[6]}$ 。 化工原理课程体系包括化工原理理论教学、化工原理实验和化工原理课程设计三个方面的课程, 三 个方面的课程都要从以教师为主的传统教学模式向以学生为主的教学方式转变。而这三类课程分别 是理论课程、实验课程和实践课程, 教学过程和教学方法都不同, 本文将分别探讨工程教育认证和 新工科建设背景下对这三门课程的课程改革方法。

\section{1 对标毕业要求, 提高化工原理理论教学水平}

化工原理理论教学是后期进行实验、实践教学环节的基础, 所以理论教学尤为重要。化工原理 课程内容的研究对象其实就是实际化学工程中的问题, 只不过是将某些化工过程中的实际问题在做 了一些合理的模型简化后进行基本原理的探讨和相关计算的研究, 然后可以直接应用研究结论对实 际工程问题进行分析描述。这种方法是基础理论课程中常用的方法, 对于培养学生由科学到技术、由 理到工的基本能力和工程问题的基本分析能力起到尤为重要的作用 ${ }^{[7]}$ 。理论教学过程中要顺应新工 科发展的需要进行适时改变, 知识结构需要不断调整, 理论知识需要不断更新, 紧跟时代步伐。总体 来讲, 要从教学内容、教学手段和方法等多方面入手, 向以学生为中心、强化工程观点, 产出导向、 持续改进转变。

\section{1 对标毕业要求, 强化课程教学目标}

我校以区域性高水平一流工科为发展定位, 培养服务地方能力突出的应用型高级专门人才。课 程要求学生具备扎实的理论知识和解决工程问题的能力和素养, 要求达到准确理解动量传递、热量 传递和质量传递的基本原理, 会运用基本原理进行相关单元操作的设计类型和操作类型计算的知识 目标; 正确评价影响单元操作的关键因素, 准确分析其对单元操作产生的影响, 掌握调控关键因素 的方法; 具有典型设备选型的能力目标和在新工科建设背景下, 掌握每个单元操作的最新发展趋势, 具有良好的协调合作沟通能力, 具备工艺工程师、设备工程师和生产工程师职业素质的素质目标。

每个专业的毕业要求需要支撑培养目标的达成, 需要明确毕业生应掌握的知识、应具备的能力 和应养成的素质。我院每个专业的毕业要求在不断完善的过程中与专业认证标准的 12 项毕业要求达 到了良好的对应与覆盖关系, 用来支撑培养目标的达成。每个毕业要求分成若干小项, 每个小项的 达成都需要培养方案中的相关课程来支撑, 根据每门课程对该毕业要求的达成支撑作用的相对大小 
制定支撑权重系数。然后, 根据每门课程在支撑毕业要求的过程中起到的作用制定课程教学目标, 以过程装备与控制工程专业的化工原理B课程为例, 在教学大纲中课程目标制定如表 1 所示。课程结 束后, 根据每个课程目标的达成情况来评价本门课程对毕业要求中的若干项的达成度。

\section{表1 课程目标对毕业要求的支撑关系}

\begin{tabular}{|c|c|c|}
\hline 毕业要求 & 毕业要求指标点 & 课程目标对毕业要求的支撑关系 \\
\hline (1) 工程知识 & $\begin{array}{l}1.4 \text { 掌握化工单元设备和成套设备的控制技术, } \\
\text { 具有对新设备、新技术进行开发与创新设计以 } \\
\text { 及项目技术经济评价的初步能力 }\end{array}$ & $\begin{array}{l}\text { 课程目标 } 1 \text { : 理解均相物系分离方法的基本原理, 掌握蒸 } \\
\text { 馏、气体吸收、干燥等单元操作的设计类型和操作类型计 } \\
\text { 算, 会运用相关计算分析单元操作中的问题。为后续化工 } \\
\text { 原理实验和化工原理课程设计课程的实践奠定理论基础 }\end{array}$ \\
\hline (2) 问题分析 & $\begin{array}{l}2.1 \text { 能通过文献研究, 运用数学、自然科学基础 } \\
\text { 知识基本原理对复杂过程装备与控制工程问题 } \\
\text { 进行识别与判断, 并结合专业知识进行关键环 } \\
\text { 节和参数的有效分解 }\end{array}$ & $\begin{array}{l}\text { 课程目标 } 2 \text { : 掌握影响蒸馏、气体吸收、干燥等单元操作 } \\
\text { 的关键因素, 会分析关键因素对单元操作产生的影响, 掌 } \\
\text { 握调控关键因素的方法 }\end{array}$ \\
\hline (3) 设计/开发解决方案 & $\begin{array}{l}3.2 \text { 具有能够根据化工单元设备和成套设备的 } \\
\text { 控制技术对新设备、新技术进行开发与创新设 } \\
\text { 计的能力 }\end{array}$ & $\begin{array}{l}\text { 课程目标 } 3 \text { : 根据每个单元操作的原理和特点, 掌握蒸馏、 } \\
\text { 气体吸收、干燥等单元操作的设计类型计算, 具备根据工 } \\
\text { 艺任务需求设计设备的能力, 为今后进行工艺设计服务 }\end{array}$ \\
\hline
\end{tabular}

\section{2 教学方法改革}

化工原理课程具有较强的工程实践性, 着重培养学生解决实际工程问题的能力。要求学生能够 灵活地将理论与实践相结合, 旨在培养学生的综合应用能力。所以, 这门课程学起来难度较大, 部 分学生对课程有畏惧心理。再者, 学生在接触这门课时往往是初涉工程领域, 缺乏理论联系实际的 能力, 普遍学习效果较差; 另一方面, 随着社会、企业对工程人才的实践能力提出更高要求, 传统 的教学模式和教学理念已经无法培养符合新标准的工程人才, 所以必须从新的教学理念出发, 在教 学方法和方式方面进行比较全面的改革。在教学方法改革方面, 结合 “两性一度” 即高阶性、创新 性、挑战度和工程教育认证的三大理念(产出导向、学生为中心、持续改进)对化工原理课程进行建 设, 将知识与学生的能力培养紧密融合, 培养学生解决复杂问题的综合能力。

原有的教学大纲内容包括教学目的、教学要求、教学内容以及讲授的时数分配等, 不能体现本 课程在毕业要求达成方面起的作用和作用大小。对原有教学大纲进行了修订, 制定明确的课程目标, 从教学内容上体现其对毕业要求达成度的支撑情况, 并制定一个合理的方案计算毕业要求达成度。 采用逆向设计-正向实施的方法, 从制定教学目标开始制定达成教学目标的测验和学习活动, 最后设 计学习活动的具体内容和策略。对于每个知识点来讲, 设计好兴趣点的安插和疑点的设置, 在适宜 处提出问题, 从而激发学生的学习兴趣, 在这整个过程中强调以学生为主体。教学设计的过程是一 个不断细化、不断改进的漫长过程。应及时更新课程内容, 将前沿知识引入课堂教学。教学形式上 要增加学生的参与度, 增加启发式教学、互动教学、案例教学等多种形式。化工原理的基本理论在 实际生产、日常生活和科学研究过程中应用都非常广泛。在备课过程中可以通过补充一些与教学内 容联系密切的案例来引导学生, 不但可以引发兴趣, 还可以锻炼学生解决实际问题的能力。合理利 用MOOC、学习通等信息化教学工具。

最后, 强化整门课程的课堂思政系统性设计, 挖掘课程体系本身蕴含的思政元素, 如科学家传 记、课程发展史、单元操作发展史等, 将其与课堂知识有机结合、融会贯通、相辅相成, 充分发挥 专业课程的 “协同育人” 功能。 


\section{3 成绩评定办法改革}

合理的成绩评定方法要遵循的指导思想是要更客观、公正、全面地体现学生的学习过程和学习 效果, 要从以结果评价为主向结果过程结合转变。一方面提高平时成绩占比, 考查学生的学习效果 的维度从单一的维度向学生的职业胜任能力、社会责任感和人文素质等多维度延伸, 不断优化平时 成绩考核方式, 使之更客观、合理, 应用信息化手段使考核过程可追溯。另一方面, 在工程教育认 证背景下, 成绩评定办法还要结合本门课程对毕业要求达成度的支撑情况制定一个方法来计算每个 学生的毕业要求达成度。同样以过程装备与控制工程专业的化工原理B课程为例来分析总评成绩的 构成和达成度的计算方法。总评成绩由平时考核成绩和期末考试成绩两部分加权而成, 平时成绩、期 末成绩及总评成绩均为百分制, 在总评成绩中, 平时成绩和期末成绩所占的权重分别为 $\alpha 1$ 和 $\alpha 2$, 具 体的成绩构成如表2所示。

\section{表2 成绩构成与其对应的课程目标}

\begin{tabular}{|c|c|c|c|c|}
\hline 课程成绩构成及比例 & 考核环节 & 目标分值 & 考核/评价细则 & 对应的课程目标 \\
\hline \multirow{10}{*}{$\begin{array}{l}\text { 平时成绩 } 100 \text { 分, 占总 } \\
\text { 评成绩的比例为 } \alpha_{1}\end{array}$} & 作业 & 25 & 主要考核学生对每章节知识点的复习、理解和掌握程度; 作业 & $1,2 、 3$ \\
\hline & & & 按章节所占学时数评分, 取各次成绩的平均值换算后作为此环 & \\
\hline & & & 节最终总成绩, 按每次作业支撑课程目标的作业评定 & \\
\hline & 单元测验 & 25 & 主要考核学生对每个单元操作知识系统掌握程度, 取各次成绩 & $1 、 2 、 3$ \\
\hline & & & 的平均值换算后作为此环节最终总成绩 & \\
\hline & 考勤 & 25 & 主要督促学生能够按时上课, 按照出勤次数占总考勤次数的比 & $1,2 、 3$ \\
\hline & & & 例折算后进入此环节最终成绩 & \\
\hline & 答疑 & 12.5 & 主要考核学生对知识点的理解和掌握程度, 对工程问题的分析 & $1,2 、 3$ \\
\hline & & & 和表达能力。答疑按次数平均后作为此环节最终成绩 & \\
\hline & 奖励 & 12.5 & 根据平时检查课堂笔记和课堂回答问题等方面给予成绩 & $1 、 2 、 3$ \\
\hline \multirow{3}{*}{$\begin{array}{l}\text { 期末考试 } 100 \text { 分, 占总 } \\
\text { 评成绩的比例为 } \alpha_{2}\end{array}$} & 课程目标1 & 40 & 卷面成绩 100 分, 以卷面成绩乘以其在总评成绩中所占的比例 & 1 \\
\hline & 课程目标2 & 20 & $\alpha_{2}$ 计入课程总评成绩 & 2 \\
\hline & 课程目标3 & 40 & & 3 \\
\hline
\end{tabular}

$\alpha_{1}+\alpha_{2}=1$, 平时成绩和期末考试成绩各自的目标分值均按 100 分设置

课程目标达成度评价包括课程分目标达成度评价和课程总目标达成度评价, 具体计算方法如下: 课程分目标达成度 $=\frac{\text { 总评成绩中支撑该课程目标相关考核环节平绩中支抣得分该课程目标相关考核环节目标总分 }}{\text { 总 }}$

课程总目标达成度 $=\frac{\text { 该课程学生总评成绩平均值 }}{\text { 该课程总评成绩总分 }(100 \text { 分 })}$

经过近两年的改革, 本人在教学工作中得到了更好的锻炼和提升, 获得校级的教学优秀奖和学 生最喜爱的教师称号; 学生学习的积极性提高, 学习效果明显提升, 以应用化学专业1班学生的化工 原理A课程成绩为例, 从2014级至2018级, 2017级和2018级的优良率相比之前有明显提升，见表3。

\section{2 化工原理实验教学内容、方式改革}

化工原理实验教学与化工原理课程的理论教学之间相辅相成, 是化工原理课程体系中非常重要 的组成部分。通过有机结合实验与理论教学有助于提高学生对课本知识的深入理解。为了进一步提 
高化工原理实验课程的教学水平, 真正通过实验培养学生的动手能力和解决实际问题的能力, 主要 从以下几个方面入手对实验课程进行改革。

表3 应用化学专业化工原理A课程成绩

\begin{tabular}{cccccc}
\hline \multirow{2}{*}{ 班级 } & \multicolumn{5}{c}{ 百分比 } \\
\cline { 2 - 6 } & $\geq 90$ 分 & $80-89$ 分 & $70-79$ 分 & $60-69$ 分 & $\leq 60$ 分 \\
\hline 应化1401 & $2 \%$ & $10 \%$ & $32 \%$ & $37 \%$ & $19 \%$ \\
应化1501 & $2 \%$ & $11 \%$ & $40 \%$ & $35 \%$ & $12 \%$ \\
应化1601 & $2 \%$ & $7 \%$ & $36 \%$ & $43 \%$ & $12 \%$ \\
应化1701 & $7 \%$ & $29 \%$ & $46 \%$ & $14 \%$ & $4 \%$ \\
应化1801 & $7 \%$ & $32 \%$ & $22 \%$ & $14 \%$ & $25 \%$ \\
\hline
\end{tabular}

\section{1 修订教学大纲, 增加实验课时, 完善实验内容}

对化工原理实验的教学大纲进行修订, 补充购买设备, 在原有的 6 个实验(离心原的性能测试及 流量计的校核、流体阻力实验、圆形直管中气体传热膜系数的测定、篎板式精馏塔的操作及塔板效 率测定、填料塔中液相传质系数的测定、流化床干燥实验)基础上新增了 4 个实验 (二氧化碳吸收与解 吸、恒压过滤常数测定、板式塔演示实验、板式换热器液液传热实验)。因为, 原有的 6 个实验不能 完全覆盖化工原理理论课中讲授的所有单元操作, 补充后实验项目更加完善, 且增加了综合性实验 的比例。目前执行的培养方案中化学工程与工艺和应用化学专业化工原理实验课程为 24 学时, 生物 工程和过程装备与控制专业为 16 学时, 学时都偏少。计划在培养方案中增加各个专业化工原理实验 的课时, 提高学生的动手、实践能力。鼓励教师和学生积极参加化工实验大赛和化工设计大赛等项 目, 以竞赛为抓手进一步促进课程建设。

\section{2 实验教学方法改革}

实验课教师的教学理念也应该进行调整, 同样要更注重培养学生独立思考和解决问题的能力, 而不是仅仅讲好实验。在教学方法上可采用对分课堂、翻转课堂等多种方式调动学生学习的热情和 探索的积极性, 引导学生形成自主学习的意识, 提高学生自主学习的能力。目前从事实验教学的教 师偏少, 新引进设备后需要组织教师积极参加培训, 承担实验教学任务。化工原理实验采用的是轮 流制的实验方法, 学生分组实验, 教师的讲解往往不可能让所有学生理解得很透彻, 不可避免得会 有学生偷懒、浑水摸鱼。为了让学生能够更加深入地理解单元操作的内容, 实验教师要学会放手 ${ }^{[8]}$, 小组内选学生自己去讲。这样, 即使是主动性比较差的学生, 为了完成讲解任务, 也会付出更多精 力和时间去先掌握单元操作的原理及过程, 可以达到事半功倍的效果。通过实验教学, 让学生巩固 理论教学的成果、提升解决工程问题的能力。

\section{3 以培养应用型人才为目标, 改革化工原理课程设计实践教学}

课程设计是整个化工原理课程体系中的最后一个环节, 其综合性和实践性相比前两个环节更强, 是考查学生使用化工原理理论课程和实验课程的知识去解决实际工程问题的一把抓手。通过化工原 理课程设计, 要求学生能综合运用本课程和前修课程的基本知识, 进行资料查阅和独立思考, 完成 指定的设计任务, 从而得到化工工程设计的初步训练 ${ }^{[9,10]}$ 。目前, 我院化工原理课程设计的主要教学 方式是教师首先集中授课, 讲解设计步骤, 然后学生参考相关资料, 按照教师制定的步骤, 进行工 艺过程计算和校核以及工艺流程及主要设备工艺条件图的绘制等工作。在课程设计中存在的问题是: 设计初始阶段, 部分学生云里雾里, 不知道从哪里入手, 后期时间紧张只能找上届同学的模板, 难 以避免抄袭现象; 设计题目难以做到一生一题, 都是分组进行, 组内同学之间难以避免抄袭现象; 
成绩评定办法太单一, 评价结果往往无法准确体现学生的真实设计水平。面对存在的问题, 对化工 原理课程设计进行了一些改革。

\section{1 课程实施前制定化工原理课程设计计划及实施方案}

为了保证课程设计的质量, 要求课程设计实施前一学期, 课程负责人制定化工原理课程设计计 划及实施方案, 具体内容包括课程设计的教学内容及基本要求、课程设计的时间、主要负责人及指 导教师安排情况、课程设计地点及安排、课程设计分组情况及指导教师负责情况、课程设计进度计 划安排、课程设计成绩评定细则、指导教师教学日志提交规范及时间要求、学生学习参考资料、归 档材料明细规范性要求等。让教师提前规划、提前准备, 从指导教师的角度保证课程设计的质量。

\section{2 课程设计成绩评定方法改革}

在以往的成绩评定中往往是综合学生的设计说明书和设计图纸来评定学生的总评成绩, 教师在 评阅的过程中, 不可能逐项去进行检查和核算, 而且对于学生之间的抄袭、抄袭往届学生设计成果 的方面, 教师很难准确把握和了解, 这就造成教师很难给出合理、公正的设计成绩。所以对成绩评 定办法进行改革很有必要, 目前执行的成绩评定办法是: 总评成绩=平时成绩 $(50 \%)+$ 设计图纸 $(20 \%)+$ 设 计说明书成绩 $(30 \%)$, 平时成绩所占的 50 分中, 考勤占 10 分, 平时设计过程中的答疑、质疑等表现(设 计表现是否积极, 是否参加设计各环节, 能否按照课程设计的要求答疑, 完成说明书的撰写、修改, 完成图纸的绘制)占20分, 交设计成果时回答老师提出的问题占 20 分。经过改革, 加强了过程性的考 核, 使得成绩更加客观和公正, 并且通过对设计过程的督导, 增强了对学生的管理, 增加了学生的 积极性和主动性。

最后, 要求指导教师在课程设计完毕后, 进行教学反思, 提交本次课程设计的总结及改进建议, 以促进对课程设计的持续改进。

\section{4 结语}

国家大力推进一流专业建设, 培养一流人才, 通过专业认证是一流本科专业建设的保障。化工 原理课程作为我院各个专业的专业基础课, 是专业认证重点关注的课程, 也是校级一流课程。所以 我们对该课程进行了比较全面的改革和探索, 使该课程真正能对各专业学生的毕业要求起到支撑作 用，并有评价的 “尺子”。

\section{参 考 文 献}

[1] 张伟光, 李金龙, 邢进, 邸凯, 辛杨, 倐白. 赵国君化工时刊, 2018, 32 (9), 53.

[2] 陈华仔, 黄双柳. 现代教育管理, 2016, No. 7,61.

[3] 赵予新. 高等工程教育质量保障研究. 北京: 社会科学文献出版社, 2014.

[4] 刘欣梅, 杨朝合, 李军, 宋春敏, 刘会娥. 中国大学教育, 2017, No. 7, 49 .

[5] 李咏梅, 周虹, 章盛祺. 高等工程教育研究, 2019, No. 6, 24 .

[6] 孙志成, 李路海, 李亚玲. 化学工程与装备, 2017, No. 7, 316 .

[7] 倪献智. 中国大学教育, 2011, No. 12, 45 .

[8] 刘建军, 徐向阳, 冯庆华, 常彦龙. 大学化学, 2016, 31 (5), 23.

[9] 肖武, 孙力, 李祥村, 贺高红, 吴雪梅, 都健, 董宏光, 潘艳秋, 韩志忠, 王瑶. 化工高等教育, 2013, No. 1, 35 .

[10] 黄英, 王艳丽, 颜红侠. 高等教育研究, 2004, 20 (3), 70. 